\title{
Propriedades físicas e químicas de substratos produzidos utilizando macrófitas aquáticas
}

\section{Physical and chemical properties of substrates produced using macrophytes aquatics}

\author{
Walda Monteiro Farias ${ }^{1 *}$; Leonaldo Alves de Andrade ${ }^{2}$; Elane Domingos Pereira ${ }^{3}$; \\ Bruno Oliveira Dias'; Manoel Bandeira de Albuquerque ${ }^{2}$; Vânia da Silva Fraga ${ }^{5}$
}

\section{Resumo}

\begin{abstract}
As macrófitas aquáticas são muito utilizadas como bioindicadoras de qualidade da água, pois sua proliferação ocorre geralmente em mananciais eutrofizados e tem atingido diversas partes do Brasil e do mundo, restringido os usos múltiplos de ecossistemas aquáticos. Contudo, esse grupo de plantas é capaz de reter considerável quantidade de nutrientes, apresentando alta produtividade e alta taxa de crescimento, constituindo assim uma boa fonte de biomassa vegetal para ser utilizada na produção de substratos. Com o objetivo de avaliar o potencial das macrófitas aquáticas aguapé (Eichhornia crassipes Solms.), alface d'água (Pistia stratiotes L.) e taboa (Typha domingensis Pers.) na produção de substratos, foi realizado, neste trabalho, sua caracterização física e química e avaliação do grau de humificação. Os tratamentos foram dispostos em esquema fatorial $3 \times 4$, delineamento inteiramente casualizado com 3 repetições. Todos os substratos produzidos com 100\% macrófita apresentam densidade dentro do limite de $400 \mathrm{~kg} \mathrm{~m}^{-3}$ considerado ideal. Os substratos compostos com alface d'água e aguapé encontram-se com condutividade elétrica de 0,79 a 2,49 $\mathrm{dS} \mathrm{m}^{-1}$, dentro do recomendado. Os compostos orgânicos produzidos são considerados maduros e apresentam elevados teores de nitrogênio fósforo e potássio; O substrato produzido com $70 \%$ alface d'água $+30 \%$ esterco e os compostos por $70 \%$ taboa $+20 \%$ esterco +10 terra vegetal e $70 \%$ taboa $+30 \%$ esterco apresentam relação $\mathrm{C} / \mathrm{N}$ dentro do considerado ideal; A razão de humificação e índice de humificação, exceto para os tratamentos 4 (70\%alface d'água $+30 \%$ esterco), 5 (100\%aguapé) e 8 (70\%aguapé+30\% esterco) encontram-se dentro do considerado ideal, a porcentagem de ácidos húmicos e índice de polimerização, exceto nos tratamentos 1(100\%alface d'água) e 12 (100\%taboa), apresentam-se abaixo do ideal.
\end{abstract}

Palavras-chave: Eichhornia crassipes, Pistia stratiotes, Typha domingensis

\footnotetext{
Abstract

Aquatic macrophytes are widely used as bioindicators of water quality because their proliferation usually occurs in eutrophic water sources and has hit several parts of Brazil and the world, restricted the multiple uses of aquatic ecosystems. However, this group of plants is able to retain considerable amounts

${ }^{1}$ Eng $^{\mathrm{a}}$ Agr $^{\mathrm{a}}$, M.e em Manejo de Solo e Água, Dept ${ }^{\mathrm{o}}$ de Solos e Engenharia Agrícola, Universidade Federal da Paraíba, Centro de Ciências Agrárias, Vila Acadêmica, UFPB, Areia, PB. E-mail: waldamonfar@yahoo.com.br

${ }^{2}$ Eng $^{\circ}{ }^{\circ}$ Agr $^{\circ \text { s }}$, Profs. Drs. do Laboratório de Ecologia Vegetal, UFPB, Centro de Ciências Agrárias, Areia, PB, Vila Acadêmica, PB. E-mail: landrade@cca.ufpb.br; mbalbuquerque@yahoo.com.br

${ }^{3}$ Enga Agra $^{\mathrm{a}}$, M.e em Manejo de Solo e Água, UFPB, Centro de Ciências Agrárias, Vila Acadêmica, Areia, PB. E-mail: elanedomingos@yahoo.com.br

${ }^{4}$ Eng $^{\circ}$ Agr $^{\circ}$, Bolsista PNPD/CAPES, Programa de Pós-Graduação em Manejo de Solo e Água, UFPB, Centro de Ciências Agrárias, Vila Acadêmica, PB. E-mail: B2dias@yahoo.com.br

${ }^{5}$ Química Industrial, Prof ${ }^{\mathrm{a}}$ Dr $^{\mathrm{a}}$ do Dept ${ }^{\mathrm{o}}$ de Solos e Engenharia Agrícola, UFPB, Centro de Ciências Agrárias, Vila Acadêmica, Areia, PB. E-mail: vfraga@cca.ufpb.br

* Autor para correspondência
} 
of nutrients, presenting high productivity and high growth rate, thus, a good source of biomass for use in the production of substrates. In order to evaluate the potential of aquatic macrophytes water hyacinth (Eichhornia crassipes Solms.), water lettuce (Pistia stratiotes L.) and cattail (Typha domingensis Pers.) in the production of substrates was performed in this work, the physical and chemical characterization and evaluation of the degree of humification. The treatments were arranged in a $3 \times 4$ factorial, completely randomized design with three replications. All substrates produced with $100 \%$ macrophyte density present within the limits of $400 \mathrm{~kg} \mathrm{~m}^{-3}$, considered ideal. The composite substrates with water hyacinth and water lettuce are with the electrical conductivity of 0,79 a $2,49 \mathrm{dS} \mathrm{m}^{-1}$ within recommended. organic compounds produced are considered mature and have high levels of nitrogen phosphorus and potassium; The substrate produced with $70 \%$ water lettuce $+30 \%$ dung and $70 \%$ composed of cattail manure $+20 \%+10 \%$ topsoil and $70+30 \%$ cattail manure have $\mathrm{C} / \mathrm{N}$ ratio within the considered ideal; the humification ratio and humification index, except for the four treatments $(70 \%$ water lettuce manure $+30 \%), 5(100 \%$ water hyacinth) and $8(70 \%$ water hyacinth manure $+30 \%)$ are within the considered ideal, the percentage of humic acids and polymerization rate, except for treatments $1(100 \%$ water lettuce) and 12 (100\% cattail), are shown below the ideal.

Key words: Eichhornia crassipes, Pistia stratiotes, Typha domingensis

\section{Introdução}

Os ambientes aquáticos são naturalmente formados por uma grande biodiversidade vegetal responsável por promover o equilíbrio e garantir o desenvolvimento desses ecossistemas. Entretanto, o crescimento desordenado dos centros urbanos, atrelado às atividades antrópicas como desmatamento, excesso de nutrientes provenientes de esgotos domésticos não tratados, poluição resultante de atividades industriais e agrícolas, bem como o processo natural de decomposição de plantas e de outros organismos promovem alterações nos corpos hídricos (PATTON; STARNES, 1970). Como resultado das diversas alterações ocorridas nestes ecossistemas pode ser observado à presença de plantas daninhas aquáticas que antes não se mostravam problemáticas.

Estas plantas aquáticas se desenvolvem desde solos saturados a ambientes completamenteaquáticos e podem ser utilizadas como bioindicadoras de qualidade de água (THOMAZ; BINI, 2003), pois, sua proliferação ocorre geralmente em mananciais eutrofizados e tem atingido diversas partes do Brasil e do mundo, restringido os usos múltiplos de ecossistemas aquáticos (VEREECKEN et al., 2006). Contudo, esse grupo de plantas é capaz de reter considerável quantidade de nutrientes, apresentam alta produtividade e alta taxa de crescimento constituindo assim, uma boa fonte de biomassa vegetal (POMPÊO; SILVA; MOSCHINICARLOS, 2005).

Plantas daninhas aquáticas, além da possibilidade de ciclagem de nutrientes que podem ser exportados via produção de substratos, possuem potencial para substituir materiais não renováveis podendo ser utilizada na produção de mudas a baixo custo, despertando assim, grande interesse nas suas propriedades fisiológicas e em seu uso potencial em benefício do homem (RUBIO et al., 2004). No entanto, para seu uso, deve-se ter conhecimento prévio das suas características, e das condições que limitam sua ocorrência e crescimento, uma vez que as macrófitas possuem capacidade de absorver elementos poluidores presentes na água, transformando-os em biomassa através da fotossíntese e a diversidade bem como produtividade destas plantas aquáticas podem ser alteradas quando as condições originais do ambiente são modificadas.

As espécies Eichhornia crassipes Solms., Pistia stratiotes L. e Typha domingensis Pers., apresentam alta capacidade de crescimento, multiplicação e facilidade de obtenção, sendo consideradas promissoras para produção de compostos orgânicos (PERAZZA; PEREIRA, MARTINS, 1981), mas devido à heterogeneidade das propriedades 
nutricionais, físicas e químicas dos materiais orgânicos, mesmo entre os provenientes da mesma origem, faz-se necessário uma caracterização inicial para utilização na composição de substratos.

Embora características físicas, como temperatura e cor do material forneçam idéia do grau de decomposição obtido, individualmente não podem estimar o estádio de maturação dos compostos orgânicos (MELO; SILVA; DIAS, 2008). Assim, métodos químicos são amplamente utilizados, incluindo análises de relação $\mathrm{C} / \mathrm{N}$, espectroscópicas, teor de $\mathrm{N}$ inorgânico e uso de índices do grau de humificação (BERNAL et al., 1998).

O objetivo deste experimento foi avaliar as características físicas e químicas, e o grau de humificação de substratos produzidos a partir das macrófitas aquáticas Eichhornia crassipes, Pistia stratiotes e Typha domingensis.

\section{Material e Métodos}

O experimento foi desenvolvido no Centro de Ciências Agrárias (CCA), Campus II, da Universidade Federal da Paraíba. Foram utilizadas as espécies Eichhornia crassipes (aguapé), Pistia stratiotes (alface d'água) e Typha domingensis (taboa). A primeira foi coletada em um lago localizado no município de Remígio-PB, as demais, em lago e solo saturado respectivamente, localizados no CCA da UFPB. Para o preparo do material, as plantas foram incorporadas a esterco bovino e terra vegetal em diferentes proporções. Os tratamentos foram dispostos em esquema fatorial $3 \times 4$, delineamento inteiramente casualizado, sendo três espécies e quatro misturas, com três repetições: macrófitas (1- Alface D’água; 2- Aguapé; 3- Taboa); substratos (1- 100\% macrófita; 2- 60\% macrófita + $30 \%$ esterco bovino $+10 \%$ terra vegetal; $3-70 \%$ macrófita $+20 \%$ esterco bovino $+10 \%$ terra vegetal; 4- 70\% macrófita $+30 \%$ esterco bovino). A parcela experimental foi representada por medas de compostagem de $1 \times 1 \times 1 m$ (comprimento $\times$ largura $\times$ altura) do material, com 5 camadas de $20 \mathrm{~cm}$, intercaladas com bambus, para facilitar a aeração, retirados 45 dias antes da coleta dos substratos do campo, que ocorreu após 150 dias da montagem do experimento. As medas foram montadas com base no material in natura.

Após coletados, os substratos foram secos ao ar livre, triturados e mensurada a biomassa para as análises realizadas em triplicata no Laboratório de Matéria Orgânica do Departamento de Solos e Engenharia Rural do CCA da UFPB. O pH foi determinado por potenciometria em uma suspensão de razão 1:5 substrato:água após as amostras serem centrifugadas por $20 \mathrm{~min}$ a $220 \mathrm{rpm}$. Condutividade elétrica foi determinada no mesmo extrato do $\mathrm{pH}$ e obtida em condutivímetro (ABREU; ANDRADE; FALCÃO, 2006). Os teores de carbono orgânico total analisados por oxidação-difusão (SNYDER; TROFYMOW, 1984); a densidade foi obtida pelo método de auto compactação (BRASIL, 2007). Para obter os teores de N, P e K total foi realizada a digestão com ácido sulfúrico e acréscimo gradativo de peróxido de hidrogênio, em bloco de digestão a $350^{\circ} \mathrm{C}$ de temperatura até obtenção da cor azul claro (THOMAS; SHEARD; MOYER, 1967), após digestão foram determinados pelo método kjeldahl (BREMNER; MULVANEY, 1982), colorimetria (MURPHY; RILEY, 1962) e por fotometria de chama, respectivamente.

Para caracterização e quantificação dos teores de C-substâncias húmicas nos substratos, foram utilizados os métodos de extração e fracionamento quantitativo de acordo com procedimento sugerido pela Sociedade Internacional de Substâncias Húmicas (IHSS) (SWIFT, 1996; MACHADO, 1999), os diferentes índices de humificação foram calculados e analisados de acordo com Jouraiphy et al. (2005): IH, ou índice de humificação, que corresponde à percentagem de $\mathrm{C}$ da fração ácido húmico $(\mathrm{C}-\mathrm{FAH})$ em relação ao $\mathrm{C}$ orgânico total (COT); RH, ou razão de humificação, que estabelece a percentagem de substâncias húmicas $(\mathrm{SH})$ em relação ao C orgânico total; \%AH, ou percentagem de ácidos húmicos, apresentado como a percentagem 
de $\mathrm{C}$ da fração ácido húmico em relação ao total das substâncias húmicas; GP, ou grau de polimerização, que representa o $\mathrm{C}$ da fração ácido húmico $(\mathrm{C}-\mathrm{AH})$ em relação ao $\mathrm{C}$ da fração ácido fúlvico $(\mathrm{C}-\mathrm{AF})$; e $\mathrm{SH}$ representa as substâncias húmicas $(\mathrm{C}-\mathrm{AH}+$ C-AF).

Os resultados foram submetidos à análise de variância com aplicação do teste $F \quad \alpha=0,05$, delineamento inteiramente casualizados, fatorial $3 \times 4$, três repetições por tratamento. Posteriormente os valores médios foram comparados entre si pelo teste de Tukey $\alpha=0,05$ com utilização do programa estatístico SAS versão 9.3(SAS, 2011).

\section{Resultados e Discussão}

$\mathrm{Na}$ Tabela 1, pode ser observado um maior teor de $\mathrm{N}$ e menor de $\mathrm{P}$ presente na terra vegetal. $\mathrm{O}$ aguapé contém os maiores teores de COT e quantidade aproximada ao esterco bovino de $\mathrm{K}$. A taboa e o aguapé apresentam alta relação $\mathrm{C} / \mathrm{N}$. Kapoor (2005), avaliando diferentes substratos para produção de compostos orgânicos, também observou altos teores de carbono para o aguapé $(41,8 \%)$.

Tabela 1. Caracterização química dos componentes dos substratos produzidos com macrófitas aquáticas.

\begin{tabular}{cccccc}
\hline COMPONENTES & $\begin{array}{c}\mathbf{N} \\
(\mathbf{g ~ k g}\end{array}$ & $\begin{array}{c}\mathbf{P} \\
\left(\mathbf{g ~ k g}^{-1}\right)\end{array}$ & $\begin{array}{c}\mathbf{K} \\
\left(\mathbf{g ~ k g}^{-1}\right)\end{array}$ & $\begin{array}{c}\mathbf{C O T} \\
\left(\mathbf{g ~ k g}^{-1}\right)\end{array}$ & $\begin{array}{c}\mathbf{C} / \mathbf{N} \\
-\end{array}$ \\
\hline Terra vegetal & 35,64 & 0,572 & 4,24 & 49,77 & 1,40 \\
Esterco bovino & 15,91 & 26,39 & 70,84 & 363,44 & 22,88 \\
Alface D'água & 24,87 & 12,11 & 35,70 & 238,24 & 9,57 \\
Aguapé & 14,89 & 11,33 & 70,24 & 614,18 & 41,44 \\
Taboa & 3,67 & 1,48 & 8,17 & 281,92 & 77,10 \\
\hline
\end{tabular}

N- nitrogênio; P-fósforo; K- potássio; COT- carbono orgânico total.

Fonte: Elaboração dos autores.

A densidade dos substratos variou de 37,34 a $707,97 \mathrm{~kg} \mathrm{~m}^{-3}$ (Figura 1), o valor considerado ideal para substratos devem ser inferiores a 400 $\mathrm{kg} \mathrm{m} \mathrm{m}^{-3}$ (ABAD; NOGUERA; BURÉS, 2001), assim a densidade dos sustratos compostos por $100 \mathrm{M}$, bem como os produzidos com $70 \mathrm{M}+30 \mathrm{E}$, exceto o composto com a taboa, encontramse com a densidade dentro do limite ideal. Os substratos $60 \mathrm{M}+30 \mathrm{E}+10 \mathrm{TV}$ e $70 \mathrm{M}+20 \mathrm{E}+10 \mathrm{TV}$ do alface d'água; $60 \mathrm{M}+30 \mathrm{E}+10 \mathrm{TV}$ do aguapé; e os $60 \mathrm{M}+30 \mathrm{E}+10 \mathrm{TV}, 70 \mathrm{M}+20 \mathrm{E}+10 \mathrm{TV}, 70 \mathrm{M}+30 \mathrm{E}$ da taboa foram superiores ao limite máximo considerado ideal, indicando que a presença da terra vegetal na composição destes eleva a sua densidade aparente, podendo dificultar o desenvolvimento e penetração de raízes.
Condutividade elétrica, variou de 0,79 a $7,45 \mathrm{dS} \mathrm{m}^{-1}$ (Figura 2). Considerando a variação recomendável para substratos 0,75 a 3,49 dS m-1 sugerida por Garcia-Gomez, Bernal e Roig (2002), apenas os substratos com 100M e 70M+30E da taboa encontram-se com CE acima do recomendado. Entretanto, Sharma et al. (1997), recomenda uma variação entre 0,64 a $6,85 \mathrm{dS} \mathrm{m}^{-1}$ para uso de resíduos na agricultura, excluindo apenas o substrato $100 \mathrm{M}$ da taboa. 
Figura 1. Densidade de substratos produzidos com macrófitas aquáticas.

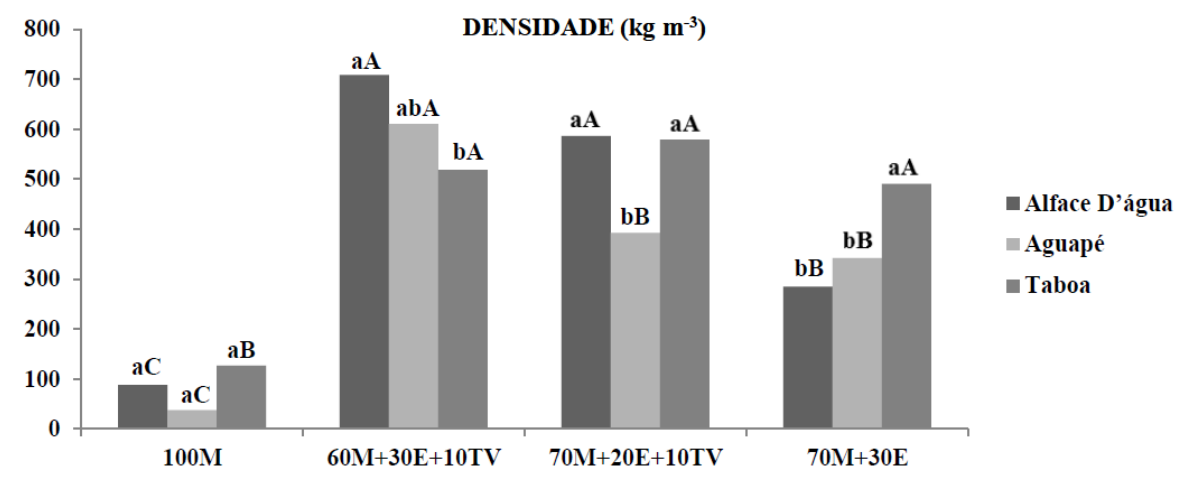

* médias seguidas da mesma letra maiúscula e minúscula não diferem entre si pelo teste de Tukey, a $\mathrm{p}>0,05 \%$.

Fonte: Elaboração dos autores.

Figura 2. Condutividade elétrica de substratos produzidos com macrófitas aquáticas.

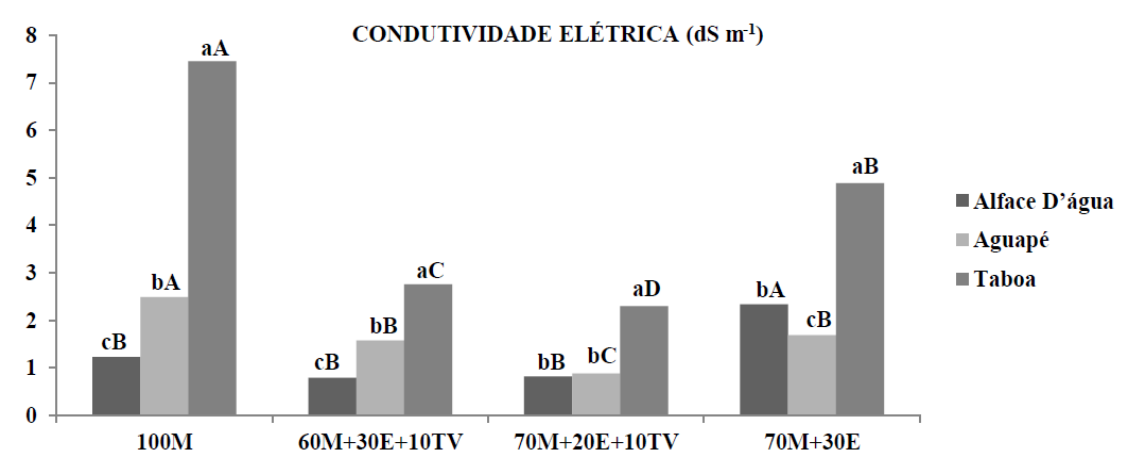

* médias seguidas da mesma letra maiúscula e minúscula não diferem entre si pelo teste de Tukey, a $\mathrm{p}>0,05 \%$.

Fonte: Elaboração dos autores.

Em relação ao COT, o substrato $70 \mathrm{M}+30 \mathrm{E}$ do alface d'água, o $100 \mathrm{M}$ e $70 \mathrm{M}+30 \mathrm{E}$ composto com aguapé e o $100 \mathrm{M}$ da taboa, apresentaram valores superiores aos demais tratamentos. Os substratos $60 \mathrm{M}+30 \mathrm{E}+10 \mathrm{TV}$ e $70 \mathrm{M}+30 \mathrm{E}$ de todas as plantas apresentaram valores semelhantes de COT. Contudo, todos os substratos são classificados como maduros segundo Bernal et al. (1998) que avaliando compostos orgânicos por 70 dias classificou como maduros substratos com teor de $\mathrm{C}<30 \%$. Cezar et al. (2005) avaliando degradação de macrófitas em ambiente protegido obteve valores de COT semelhantes aos tratamentos compostos por $100 \%$ das macrófitas e aos com 70\% macrófitas $+30 \%$ de esterco, diferindo dos substratos $60 \mathrm{M}+30 \mathrm{E}+10 \mathrm{TV}$ e $70 \mathrm{M}+20 \mathrm{E}+10 \mathrm{TV}$ que apresentam terra vegetal em sua composição e menor teor de COT (Figura 3), o que pode ser explicado pelo baixo teor de carbono presente na terra vegetal quando comparado à planta e ao esterco bovino (Tabela 1). 
Figura 3. Carbono orgânico total de substratos produzidos com macrófitas aquáticas.

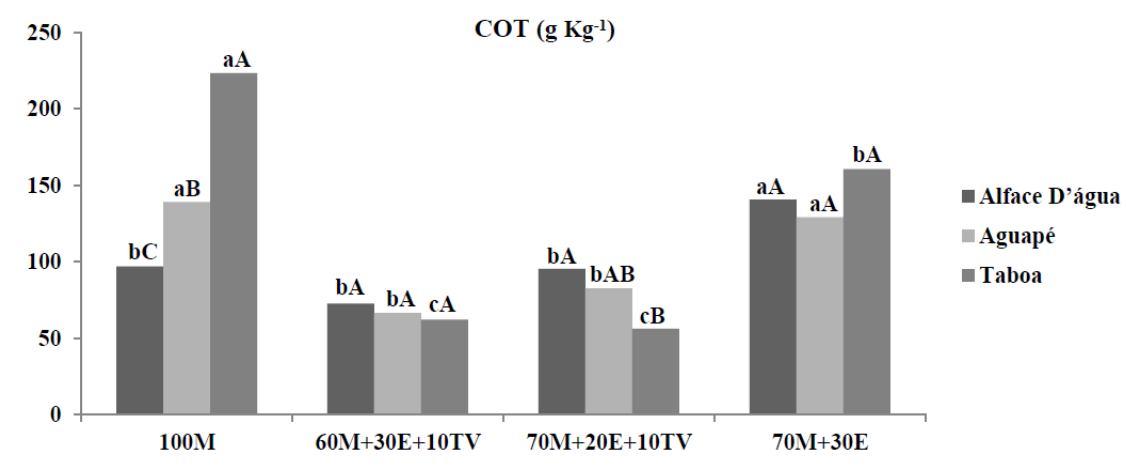

* médias seguidas da mesma letra maiúscula e minúscula não diferem entre si pelo teste de Tukey, a $\mathrm{p}>0,05 \%$.

Fonte: Elaboração dos autores.

Não houve interação significativa entre as ideal para os substratos utilizados na produção de macrófitas e os substratos para avaliação do $\mathrm{pH}, \mathrm{N}$, mudas em recipientes seria 5,3-6,5. No entanto os $\mathrm{P}$ e K, com aplicação do teste $\mathrm{F} \alpha=0,05$ e $\mathrm{p}>0,05 \%$. substratos produzidos com as macrófitas poderiam $\mathrm{O}$ pH de todos os substratos encontra-se alcalino ser utilizados, tendo em conta que a acidez final do (Figura 4), associado a influência do esterco bovino substrato pode ser ajustada. De acordo com Silva et que apresentou pH 9,2. De acordo com Abad, al. (1997), a faixa de $\mathrm{pH}$ permitida dos resíduos para Noguera e Burés (2001), a faixa de pH considerada uso agrícola é 6,0-8,5.

Figura 4. pH de substratos produzidos com macrófitas aquáticas.
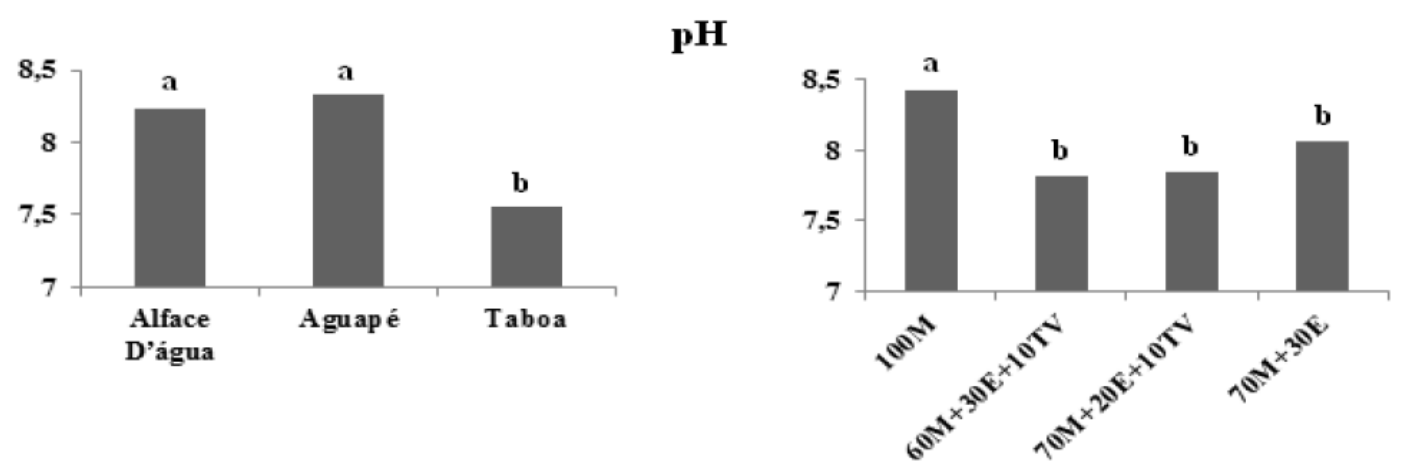

* médias seguidas da mesma letra maiúscula e minúscula não diferem entre si pelo teste de Tukey, a p >0,05\%.

Fonte: Elaboração dos autores.

A taboa apresentou os menores teores de $\mathrm{N}$ e P total, o que pode ser explicado pela baixa concentração destes elementos na planta, (Figuras 5 e 6), teores semelhantes de $\mathrm{P}$ e superiores de $\mathrm{N}$ foram encontrados por Sousa (2003) em estudo avaliando Thypha domingensis no tratamento de efluentes. O substrato composto por $100 \%$ macrófita apresentou os maiores teores de $\mathrm{N}$ total. Cezar et al. (2005) avaliando degradação de macrófitas em ambiente protegido obteve teores semelhantes de N. Não houve diferença do teor de P total entre os substratos. 
Figura 5. Nitrogênio de substratos produzidos com macrófitas aquáticas.

N
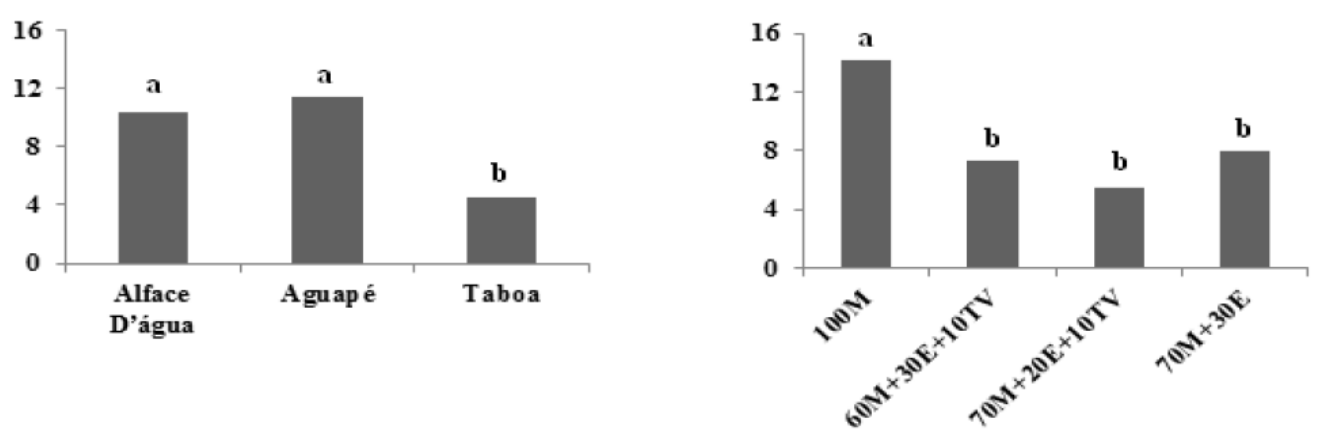

* médias seguidas da mesma letra maiúscula e minúscula não diferem entre si pelo teste de Tukey, a p >0,05\%.

Fonte: Elaboração dos autores.

Figura 6. Fósforo de substratos produzidos com macrófitas aquáticas.

\section{$\mathbf{P}$}
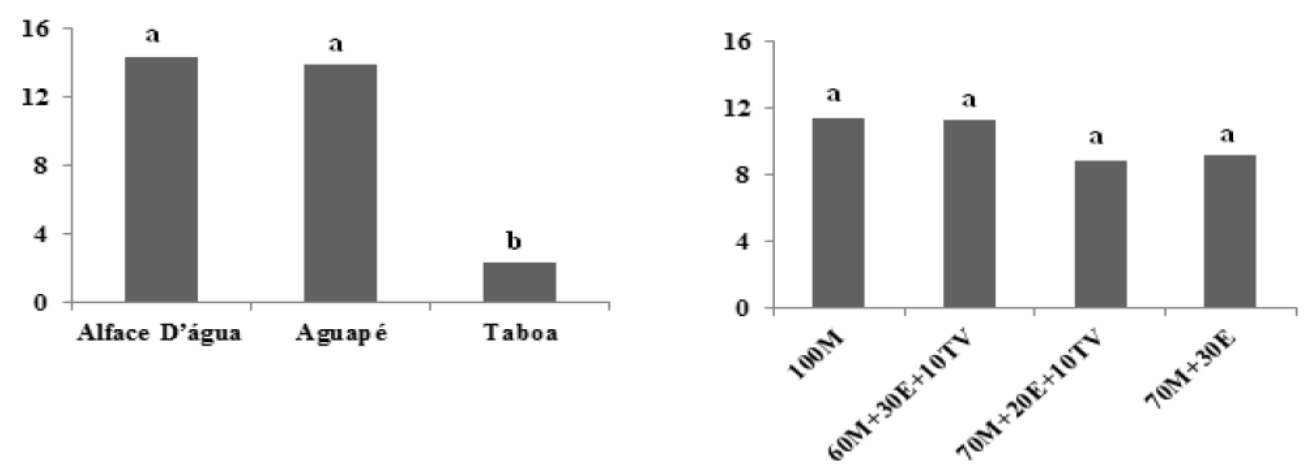

* médias seguidas da mesma letra maiúscula e minúscula não diferem entre si pelo teste de Tukey, a $\mathrm{p}>0,05 \%$.

Fonte: Elaboração dos autores.

A concentração de potássio não diferiu entre alface d'água e taboa. O aguapé apresentou teores superiores de $\mathrm{K}\left(16,26 \mathrm{mg} \mathrm{g}^{-1}\right)$, assim como o substrato $100 \mathrm{M}\left(16,41 \mathrm{mg} \mathrm{g}^{-1}\right)$ figura 7 . Os teores de fósforo, nitrogênio e potássio encontrados são considerados altos (KIEHL, 1985) e encontram-se semelhantes aos teores observados em estercos em pesquisa com comparação de métodos de digestão de resíduos orgânicos (MELO, 2007).

Não houve diferença entre o AH dos substratos do alface d'água e aguapé (Figura 8). O substrato 100M da taboa apresentou maior teor de $\mathrm{AH}$, demostrando um menor nível de decomposição, uma vez que quanto maior o teor de $\mathrm{AH}$ menos humificado se encontra o material. Os substratos $70 \mathrm{M}+20 \mathrm{E}+10 \mathrm{TV}$ e $70 \mathrm{M}+30 \mathrm{E}$ do alface d'água e $100 \mathrm{M}$ do aguapé apresentaram teores de AF superiores aos demais (Figura 9). Não houve diferença estatística entre os teores de AF dos materiais produzidos com a taboa. Os substratos 100M do aguapé, do $60 \mathrm{M}+30 \mathrm{E}+10 \mathrm{TV}$ do alface d'água e aguapé, $70 \mathrm{M}+20 \mathrm{E}+10 \mathrm{TV}$ do alface d'água e do $70 \mathrm{M}+30 \mathrm{E}$ do alface d'água e aguapé foram superiores aos demais. 
Figura 7. Potássio de substratos produzidos com macrófitas aquáticas
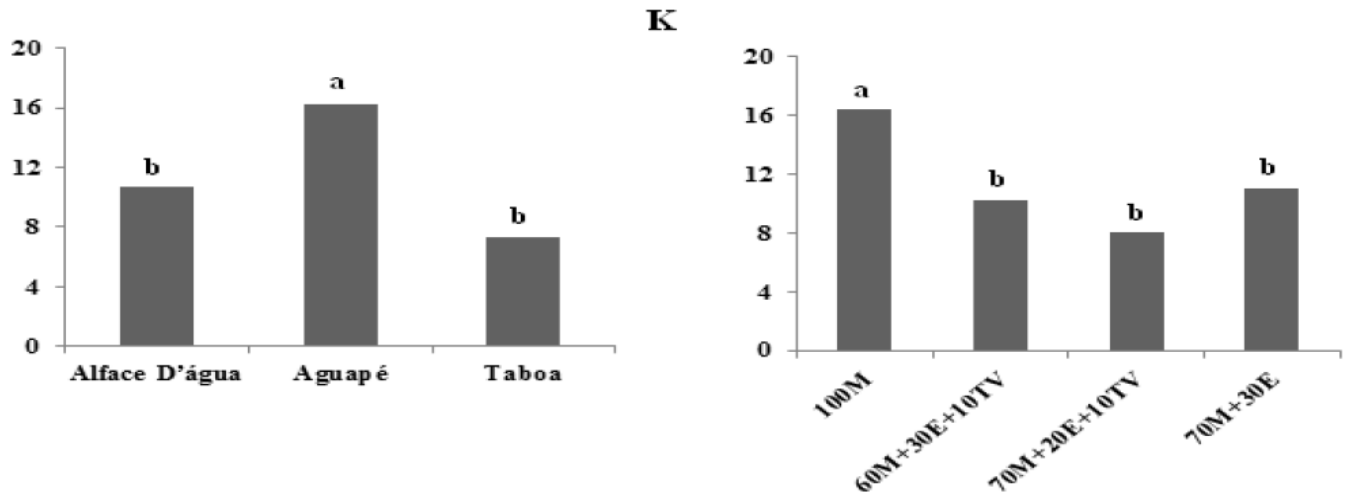

* médias seguidas da mesma letra maiúscula e minúscula não diferem entre si pelo teste de Tukey, a $\mathrm{p}>0,05 \%$.

Fonte: Elaboração dos autores.

Figura 8. Ácido húmico de substratos produzidos com macrófitas aquáticas.

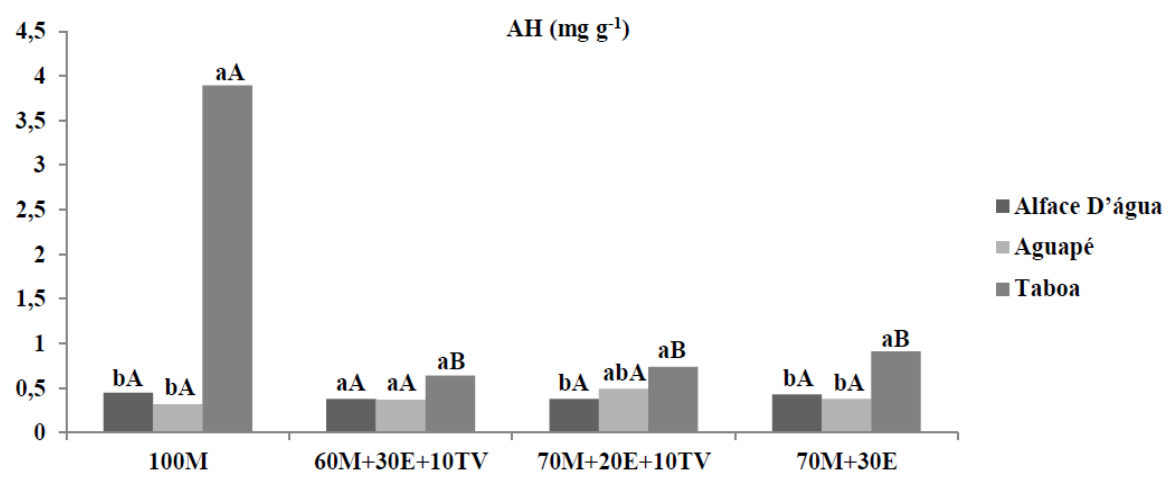

* médias seguidas da mesma letra maiúscula e minúscula não diferem entre si pelo teste de Tukey, a p $>0,05 \%$.

Fonte: Elaboração dos autores.

Figura 9. Ácido fúlvico de substratos produzidos com macrófitas aquáticas

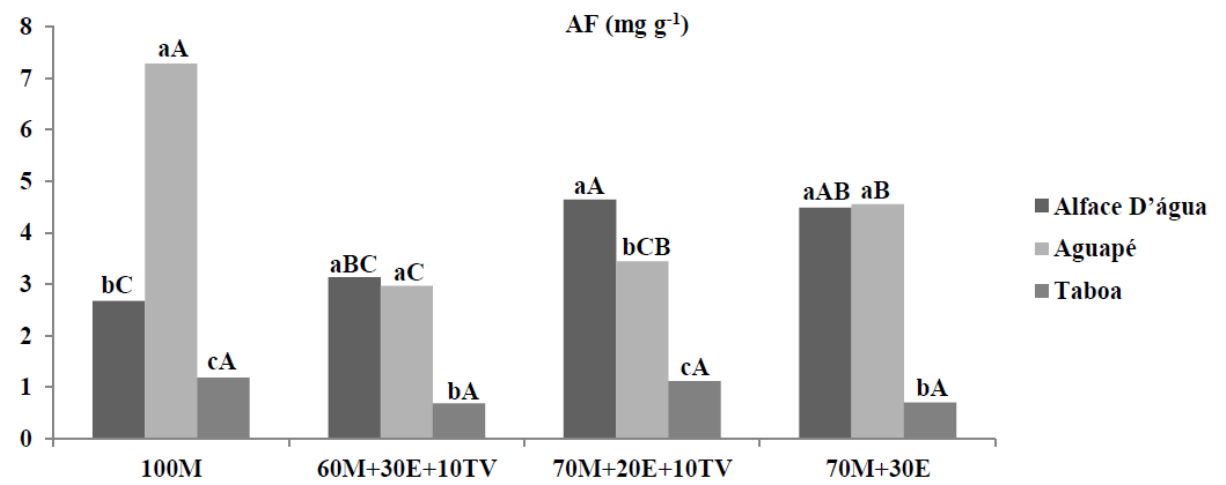

* médias seguidas da mesma letra maiúscula e minúscula não diferem entre si pelo teste de Tukey, a $\mathrm{p}>0,05 \%$.

Fonte: Elaboração dos autores. 
Os teores de AF foram maiores que os de $\mathrm{AH}$, exceto no substrato $100 \mathrm{M}$ e $70 \mathrm{M}+30 \mathrm{E}$ da taboa, esses valores encontram-se inferiores aos encontrados por Moral et al. (2005) em estudo de caracterização de matriz orgânica de estercos de diversas origens, porém neste mesmo trabalho o autor também observou a predominância de C-ácido fúlvico sobre o $\mathrm{C}$-ácido húmico na maioria dos estercos, o que indica que a matéria orgânica desses substratos não se encontra em estágio avançado de humificação. Durante o processo de compostagem há um incremento nos teores de substâncias húmicas ocorrendo uma redução nos teores da fração ácido fúlvico e um aumento da fração ácido húmico. Sendo caracterizado um composto já estabilizado e humificado o que apresenta uma maior proporção da fração ácido húmico (BERNAL; ALBURQUERQUE; MORAL, 2009).

O substrato 4 (M1S4) produzido com o alface d'água e os compostos 11 e 12 (M3S3 e M3S4) produzidos com a macrófita Typha domingensis apresentaram relação $\mathrm{C} / \mathrm{N}$ adequada (Figura 10).
Segundo Pereira Neto (1996), torno de 30:1, e (KIEHL, 1998) de 25:1 a 30:1. Porém ao considerar o valor recomendado por Brasil (2011) de 20:1, a relação dos substratos produzidos com a macrófita I (exceto S4) e macrófita II encontra-se baixa e da macrófita III (exceto M3S2) encontra-se alto. Resíduos orgânicos com relação $\mathrm{C} / \mathrm{N}$ alta, podem provocar imobilização temporária de $\mathrm{N}$ pelos microrganismos. Segundo Bernal et al. (1998), a adição de materiais com relação $\mathrm{C} / \mathrm{N}$ abaixo de 15 não altera o equilíbrio microbiológico, não havendo, portanto, imobilização de $\mathrm{N}$.

A avaliação do grau de humificação da matéria orgânica durante a compostagem é um critério importante para analisar a qualidade dos compostos. A figura11 apresenta respectivamente os índices razão de humificação $(\mathrm{RH})$ e índice de humificação (IH). Pode ser observado, exceto nos tratamentos 4 (M1S4), 5(M2S1), 8(M2S4), que os substratos analisados apresentam-se humificados (valores $\geq$ 7,0 e $\geq 3,5$ respectivamente) Roletto et al. (1985).

Figura 10. Relação $\mathrm{C} / \mathrm{N}$ de substratos produzidos com macrófitas aquáticas.

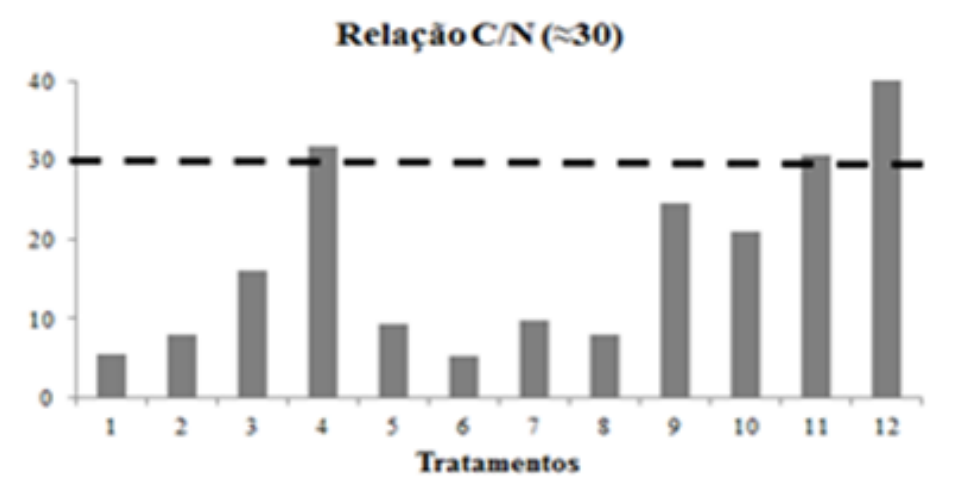

Tratamentos: 1(M1S1), 2(M1S2), 3(M1S3), 4(M1S4), 5(M2S1), 6(M2S2), 7(M2S3), 8(M2S4), 9(M3S1), 10(M3S2), 11(M3S3), 12(M3S4).

Fonte: Elaboração dos autores. 
Figura 11. Razão e índice de humificação de substratos produzidos com macrófitas aquáticas.
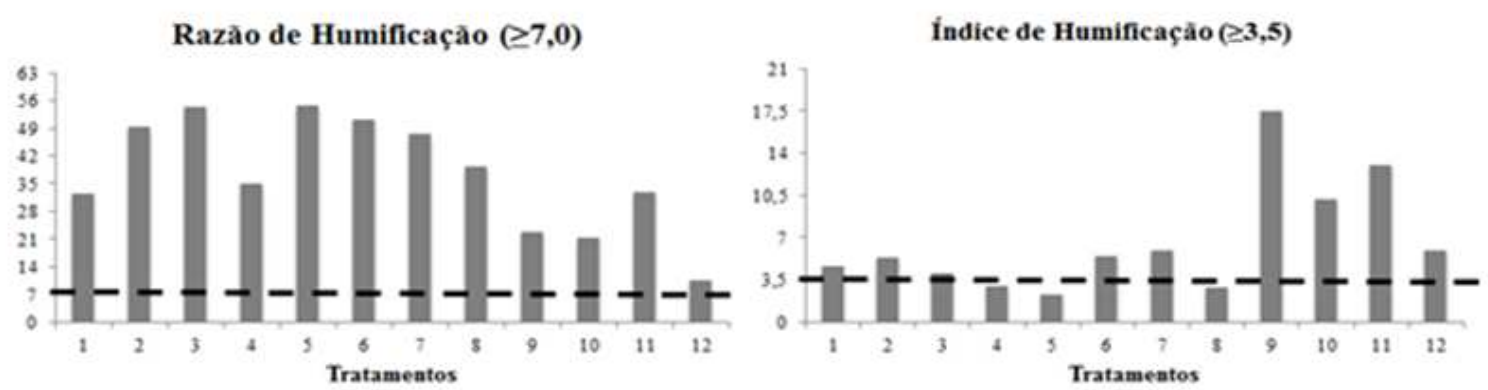

Tratamentos: 1(M1S1), 2(M1S2), 3(M1S3), 4(M1S4), 5(M2S1), 6(M2S2), 7(M2S3), 8(M2S4), 9(M3S1), 10(M3S2), 11(M3S3), 12(M3S4).

Fonte: Elaboração dos autores.

Figura 12. Porcentagem de ácidos húmicos e Índice de polimerização de substratos produzidos com macrófitas aquáticas.
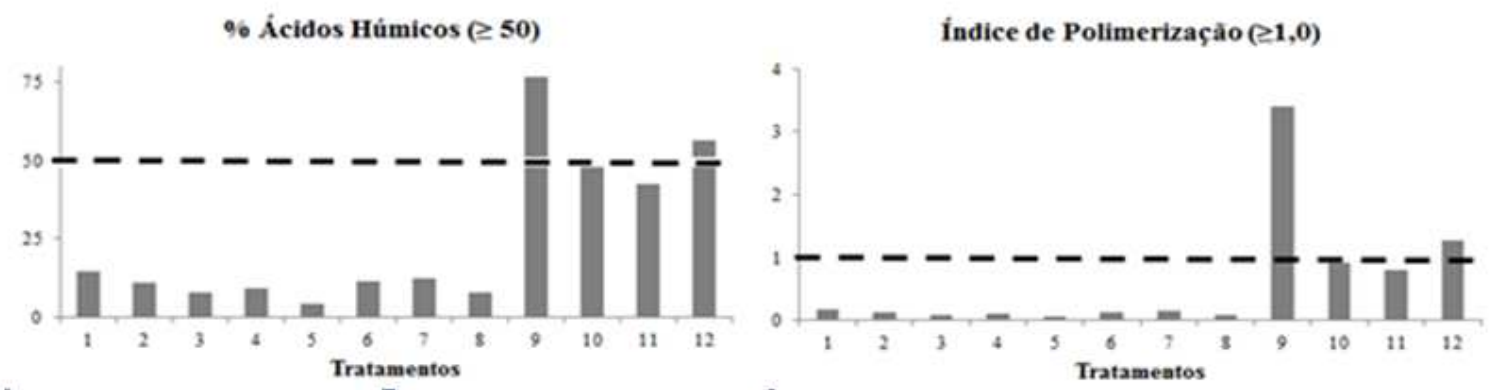

Tratamentos: 1(M1S1), 2(M1S2), 3(M1S3), 4(M1S4), 5(M2S1), 6(M2S2), 7(M2S3), 8(M2S4), 9(M3S1), 10(M3S2), 11(M3S3), 12(M3S4).

Fonte: Elaboração dos autores.

Dos valores de porcentagem de ácido húmico e índice de polimerização (figura 12) observase que os tratamentos apresentam valores abaixo do considerado ideal, com exceção do tratamento 1 (M3S1) e do 12 (M3S4) que de acordo com os limites estipulados por Roletto et al.,(1985) para os índices de humificação encontram-se humificados.

Foi efetuado o monitoramento da temperatura média interna das pilhas de compostagem, observando-se um aumento inicial de temperatura em todos os tratamentos, entretanto, mantiveramse entre $20^{\circ}$ e $30^{\circ} \mathrm{C}$ (Figura 13), ficando abaixo do ideal para o processo de decomposição. Segundo Kiehl (1998) a temperatura, principalmente na etapa que vai de 0 a 30 dias, deve ser de até $55^{\circ} \mathrm{C}$. Provavelmente este fato tenha ocorrido pela elevada aeração no interior das leiras ocasionadas pela presença dos bambus, combinada ao tamanho das partículas submetidas à decomposição, pois para montagem das medas de compostagem as plantas e demais materiais utilizados não passaram por nenhum processo de trituração prévia. Mees (2009) observou as mesmas condições em estudo com compostagem de aguapé. 
Figura 13. Temperatura média de substratos produzidos com macrófitas aquáticas.

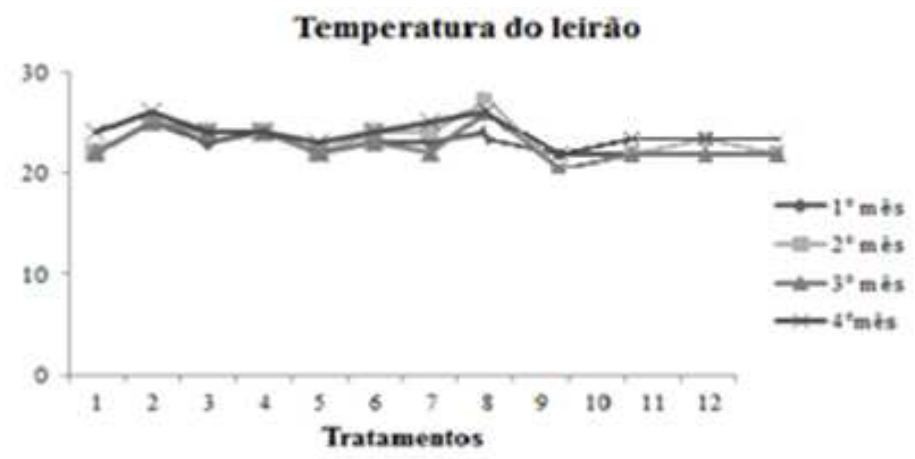

Tratamentos: 1(M1S1), 2(M1S2), 3(M1S3), 4(M1S4), 5(M2S1), 6(M2S2), 7(M2S3), 8(M2S4), 9(M3S1), 10(M3S2), 11(M3S3), 12(M3S4).

Fonte: Elaboração dos autores.

A utilização de macrófitas aquáticas na composição de substratos é uma fonte alternativa viável e com características nutricionais semelhantes à de compostos orgânicos comumente utilizados na agricultura, podendo ser utilizado como substrato alternativo na produção de mudas. Porém, estudos adicionais são necessários para obter maior acurácia sobre a utilização destes substratos.

\section{Conclusões}

Os compostos produzidos com as espécies estudadas constituem boa fonte de N, P e K, exceto o composto por $100 \%$ da Typha domingensis Pers. e apresentam densidade e condutividade elétrica dentro do recomendado.

\section{Agradecimentos}

A CAPES e CNPq.

\section{Referências}

ABAD, M.; NOGUERA, P.; BURÉS, S. National inventory of organic wastes for use as growing media for ornamental potted plant production: case study in Spain. Bioresourse Technology, New York, v. 77, n. 2, p. 197200, 2001.
ABREU, M. F.; ANDRADE, J. C.; FALCÃO, A. A. Protocolos de análises químicas. In: ANDRADE, J. C.; ABREU, M. F. (Org.). Química de resíduos sólidos para monitoramento e estudos agroambientais. Campinas: Instituto Agronômico de Campinas, 2006. p. 121-158.

BERNAL, M. P.; ALBURQUERQUE, J. A.; MORAL, R. Composting of animal manures and chemical criteria for compost maturity assessment. A review. Bioresource Technology, New York, v. 100, n. 5, p. 444-5453, 2009.

BERNAL, M. P.; PAREDES, C.; SÄNCHEZ MONEDERO, M. A.; CEGARRA, J. Maturity and stability parameters of composts prepared with a wide range of organic wastes. Bioresourse Technology, New York, v. 63, n. 1, p. 91-99, 1998.

BRASIL. Ministério da Agricultura, Pecuária e Abastecimento. Instrução Normativa $n^{\circ} 17$, de 21 de maio de 2007. Aprova os métodos analíticos oficiais para análise de substratos e condicionadores de solos, na forma do anexo à presente instrução normativa. Diário Oficial [da] União da República Federativa do Brasil, Brasília, 24 maio 2007. Seção 1, p. 8.

Instrução Normativa $n^{\circ} 25$, de 23

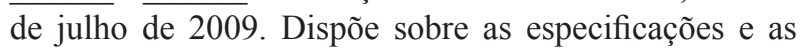
garantias, as tolerâncias, o registro, a embalagem e a rotulagem dos fertilizantes orgânicos simples, mistos, compostos, organominerais e biofertilizantes destinados à agricultura. Diário Oficial [da] União, Brasília, 28 de julho de 2009. Seção 1, p. 20. Disponível em: <http:// www.agricultura.gov.br>. Acesso em: 14 dez. 2011. 
BREMNER, J. M.; MULVANEY, C. S. Nitrogen-total. In: PAGE, A. L.; MILLER, R. H.; KEENEY, D. R. (Ed.). Methods of soil analysis. Chemical and microbiological properties. Part 2. Madison: ASA-SSSA, 1982. p. 595624.

CEZAR, V. R. S.; VILLAS BOAS, R. L.; CORRÊA, M. R.; NEGRISOLI, E.; VELINI, E. D. Avaliação da degradação de macrófitas aquáticas descartadas em ambiente protegido. Planta Daninha, Viçosa, MG, v. 23, n. 2, p. 255-261, 2005.

GARCIA-GOMEZ, A.; BERNAL, M. P.; ROIG, A. Growth of ornamental plants in two composts prepared from agroindustrial wastes. Bioresour Technology, New York, v. 83, n. 2, p. 81-87, 2002.

JOURAIPHY, A.; AMIR, S.; EL GHAROUS, M.; EVEL, J.; HAFIDI, M. Chemical and spectroscopic analysis of organic matter transformation during composting of ewage sludge and green plant waste. International Biodeterioration \& Biodegradation, New York, v. 56, n. 2, p. 101-108, 2005.

KAPOOR, K. K.; GOYAL, S.; DHULL, S. K.; KAPOOR, K. K. Chemical and biological changes during composting of different organic wastes and assessment of compost maturity. Bioresource Technology, Essex, v. 96, n. 14, p. 1584-1591, 2005.

KIEHL, E. J. Fertilizantes orgânicos. São Paulo: Agronômica Ceres, 1985. 492 p.

MACHADO, P. L. O. Método para a extração de substâncias húmicas do solo: ácido - húmico e ácido fúlvico. Rio de Janeiro: Embrapa Solos, 1999. 4 p. (Comunicado técnico, 1).

MEES, J. B. R.; DAMASCENO, S.; VILAS BOAS, M. A.; FAZOLO, A.; SAMPAIO, S. C. Estabilização da biomassa de aguapé através da compostagem com águas residuárias de suínos e resíduos de frigorífico. Semina: Ciências Agrárias, Londrina, v. 30, n. 3, p. 709-716, 2009.

MELO, L. C. A. Caracterização físico-química e comparação de métodos de digestão de resíduos agrícolas. 2007. Dissertação (Mestrado em Agronomia) - Universidade Federal de Lavras, Lavras.

MELO, L. C. A.; SILVA, C. A.; DIAS, B. O. Caracterização da matriz orgânica de resíduos de origens diversificadas. Revista Brasileira de Ciência do Solo, Viçosa, MG, v. 32, n. 1, p. 101-110, 2008.

MORAL，R.; MORENO-CASELLES, J.; PERREZMURCIA, M. D.; PEREZ-ESPINOSA, A.; RUFETE, B.; PAREDES, C. Characterization of the organic matter pool in manures. Bioresource Technology, New York, v. 96, n. 2, p. 153-158, 2005.
MURPHY, J.; RILEY, J. P. A. A modified simple solution method for the determination of phosphate in natural waters. Analytica Chimica Acta, New York, v. 27, p. 3136, 1962.

PATTON, V. D.; STARNES, W. E. Aquatic weeds and water pollution. Hyacinth Control Journal, Florida, v. 8, n. 2, p. 48-49, 1970.

PERAZZA, M. C. D.; PEREIRA, D. N.; MARTINS, M. T. O aguapé: meios de controle e possibilidades de utilização. Revista DAE, São Paulo, n. 125, p. 18-24, 1981.

PEREIRA NETO, J. T. Conceitos modernos de compostagem. Revista de Engenharia Sanitária, Rio de Janeiro, v. 1, n. 1, p. 1-6, abr.jun. 1989. (Manual de compostagem: processo de baixo custo. Belo Horizonte: UNICEF, 1996).

POMPÊO, M. L.; SILVA, S. C.; MOSCHINI-CARLOS, V. A deterioração da qualidade das águas continentais brasileiras: o processo de eutrofização. Saneas, São Paulo, v. 21, n. 2, p. 24-28, 2005.

ROLETTO, E.; BARBERIS, R.; CONSIGLIO, M.; JODICE, R. Chemical parameters for evaluating compost maturity. Biocycle, Ohio, v. 26, n. 2, p. 46-47, mar. 1985.

RUBIO, J.; SCHNEIDER, I. A. H.; RIBEIRO, T.; COSTA, C. A.; KALlFEZ, C. A. Plantas aquáticas: sorventes naturais. Ciência hoje, Recife, v. 35, n. 205, jun. 2004.

STATISTICAL ANALYSIS SYSTEM INSTITUTE - SAS. SAS/TAT 9.3. User's guide. Cary, NC: SAS Institute Inc., 2011. 8621 p.

SHARMA, V. K.; CANDITELLI, M.; FORTUNA, F.; CORNACCHIA, G. Processing of urban and agroindustrial residues by aerobic composting. Rev. Energy Conserv. Manag., Oxford, v. 38, n. 5, p. 453-478, 1997.

SILVA, M. S.; MENDONÇA, C. L. A.; VILAS BOAS, M. A.; SILVA, M. S. Compostagem de resíduos sólidos de frigorífico, visando o saneamento ambiental e a produção de adubo orgânico. In: CONGRESSO BRASILEIRO DE CIÊNCIA DO SOLO, 26., 1997, Rio de Janeiro. Anais... Rio de Janeiro: Agronômica Ceres, 1997. CD-ROM.

SNYDER, J. D.; TROFYMO-W, J. A. A rapid accurate wet oxidation diffusion procedure for determining organic and inorganic carbon in plant and soil samples. In Comm. Soil Sci. Plant Anal., New York, v. 15, n. 5, p. 587-597, 1984. 
SOUSA, L. M. I. Avaliação da macrófita Thypha Domingensis Pers. no pós-tratamento de efluentes do campus da UFMS e do hospital universitário, em banhados construidos de fluxo subsuperficial. 2003. Dissertação (Mestrado em Agronomia) - Universidade Federal de Mato Grosso do Sul, Chapadão do Sul.

SWIFT, R. S. Organic matter characterization. In: SPARKS, D.; PAGE, A. L.; HELMKE, P. A.; LOEPPERT, R. H.; SOLTANPOUR, P. N.; TABATABAI, M. A.; JOHNSTON, C. T.; SUMMER, M. E. (Ed.). Methods of soil analysis. Madison: Soil Science Society of America/ American Society of Agronomy, 1996. Part 3, p. 10111020.
THOMAS, R. L.; SHEARD, R. W.; MOYER, J. R. Comparison of conventional and automated procedures for nitrogen, phosphorus and potassium analysis of plant material using single digest. Agron. J., Madison, v. 59, n. 3, p. 240-243, 1967.

THOMAZ, S. M.; BINI, L. M. Ecologia e manejo de macrófitas aquáticas. Maringá: UEM, 2003. 341 p.

VEREECKEN, V.; BAETENS, J.; VIAENE, P.; MOSTAERT, F.; MEIRE, P. Ecological management of aquatic plants: effects in lowland streams. Hydrobiologia, New York, v. 570, n. 1, p. 205-210, 2006. 
